\title{
LIDAR and Beam Steering Tailored by Neuromorphic Metasurfaces Dipped in a Tunable Surrounding Medium
}

\author{
Giuseppe Emanuele Lio (D) and Antonio Ferraro *(D) \\ CNR-Nanotec and Department of Physics, University of Calabria, 86037 Rende, Cosenza, Italy; \\ giuseppe.lio@unical.it \\ * Correspondence: antonio.ferraro@unical.it
}

check for

updates

Citation: Lio, G.E.; Ferraro, A. LIDAR and Beam Steering Tailored by Neuromorphic Metasurfaces Dipped in Tunable Surrounding Medium. Photonics 2021, 8, 65. https: / / doi.org/10.3390/ photonics 8030065

Received: 2 February 2021

Accepted: 23 February 2021

Published: 26 February 2021

Publisher's Note: MDPI stays neutral with regard to jurisdictional claims in published maps and institutional affiliations.

Copyright: (c) 2021 by the authors. Licensee MDPI, Basel, Switzerland. This article is an open access article distributed under the terms and conditions of the Creative Commons Attribution (CC BY) license (https:// creativecommons.org/licenses/by/ $4.0 /)$.

\begin{abstract}
The control of amplitude, losses and deflection of light with elements of an optical array is of paramount importance for realizing dynamic beam steering for light detection and ranging applications (LIDAR). In this paper, we propose an optical beam steering device, operating at a wavelength of $1550 \mathrm{~nm}$, based on high index material as molybdenum disulfide $\left(\mathrm{MoS}_{2}\right)$ where the direction of the light is actively controlled by means of liquid crystal. The metasurface have been designed by a deep machine learning algorithm jointed with an optimizer in order to obtain univocal optical responses. The achieved numerical results represent a promising way for the realization of novel LIDAR for future applications with increase control and precision.
\end{abstract}

Keywords: beam steering; tunable devices; metasurfaces; deep machine learning

\section{Introduction}

The advantages provided by new technologies are widely and extremely important for applications such as optics for portable devices, imaging, signal detection, and Light Detection and Ranging or Laser Imaging Detection and Ranging (LIDAR). In order to correctly introduce this technology and its wide scientific interest, it is necessary breifly discuss its history. Everything began thanks to Theodore Maiman who, in the 1960s, built and demonstrated the first practical laser. Successively, Massachusetts Institute of Technology (MIT) scientists measure the distance between Earth and the Moon using a reflected laser beam, and solely in 1965, Stanford Research Institute's Ronald Collins filed a patent for a laser-radar LIDAR system that can be used to study Earth's atmosphere and weather. The first prototype of the LIDAR system has been involved in 1971 by Apollo 15 to map the surface of the moon, in 1976, the first textbox of that system has been published, in the 1990s it becomes widely used in the geographical mapping. In the last two decades, several applications have been developed: in 2005 LIDAR systems make the headlines as the eyes behind self-driving cars in the US military's DARPA Grand Challenge, in 2008 NASA's Phoenix Lander bring an Optech LIDAR scanner to Mars for studying the planet's atmosphere. Finally, in 2020, a LIDAR has been included in a smartphone to improve 3D modeling of the environment around it for augmented reality applications [1-4]. In order to increase the precision and image harvesting of these systems, many efforts have been done by the scientific community in the study of beam steering. The beam steering was addressed in several different approaches, such as using nanodevices, metamaterials, metastructures and metasurfaces, liquid crystals, just to name a few [5-12]. In particular, the refractive index of LC can be easily controlled by external stimuli as electric/magnetic filed, or temperature and can be easily integrated into practical devices by using display technology [13-15]. The design of structures able to control the light beam is daily addressed by the use of sophisticated numerical systems approaches that exploit artificial intelligence and deep machine learning algorithms. These approaches give a further leap forward in the numerical design of metasurfaces for heat 
generation, for plasmonic chiral response and light detection, for sensors, internet of thing, and so on [16-21].

The basic principle of the case study is based on the combination of an on-chip switch/emitter structure as already addressed in literature [22]. The light beam, propagated and modulated from the metastructures, intersects at one point the scanning plane of the device meaning that beam-steering can be easily achieved by selecting the refractive index of the cover medium $(n)$. According to the Fourier optics theory, the beam pattern near the scanning point (sp), i.e., $E_{\text {out } 1}(x, y)$, is the Fourier transform $(F)$ of the beam pattern on the emitter array, which is expressed as:

$$
E_{\text {out } 1}(x, y)=-\left.\frac{i}{\lambda \cdot s p \cdot n} F\left[E_{i n}\left(x_{0}, y_{0}\right)\right]\right|_{f_{x}=\frac{x}{\lambda s p}, s p_{y}=\frac{y}{\lambda s p}}
$$

where $E_{\text {in }}(x 0, y 0)$ is the beam that impinges on the metastructures array and $\lambda$ is its wavelength. Therefore, the far field beam intensity pattern is fully determined by the beam pattern in the metastructure array plane. Meanwhile, beam divergence angle $\Delta \theta$ is approximately expressed as follows[23]:

$$
\Delta \theta=\tan ^{-1}\left(\frac{w}{s p \cdot n}\right)
$$

where $w$ is the near-field beam diameter. The beam-steering angle $\theta$ is approximately given by [23]:

$$
\theta=\tan ^{-1}\left(\frac{l}{s p \cdot n}\right)
$$

where $l$ is the length of each metastructure array. These equations indicate that the beam divergence and beam-steering angle can be engineered by properly choosing the beam diameter of the source, the metasurfaces size and spacing, the surrounding refractive index and the distance of the scanning plane. The devices using beam steering for LIDAR applications could be passive or active systems. In the former case, they harvest part of the sunlight into a detector and use the beam bending to redefine the observed objects or surfaces. In the active case, a diode emits light that passes through the device and it is deflected in all directions and finally, as a result, the beam comes back in the detector reconstructing the final image [24-26].

The use of wavelength at $1550 \mathrm{~nm}$ in beam steering and LIDAR application find a wide interest thanks to the variety of miniaturized source available at that frequency, a long-range and better resolution with a diffraction-limited output beam and is most importantly eye-safe, also ensuring use in commercial cameras and smartphones [27]. In this work we numerical design, by inverse design mediated by deep machine learning, a device able to drive the light in the function of external stimuli reaching a beam steering of $20^{\circ}$ at the telecommunication wavelength of $(\lambda=1550 \mathrm{~nm})$. The proposed device is composed of a metasurface of high refractive index materials such as molybdenum disulfide $\left(\mathrm{MoS}_{2}\right)$ covered with a new class of liquid crystals (LCs) exhibiting an high birefringence $(\Delta n=0.4)$ [28]. The compact and low voltage operation of the investigated device can be easily integrated in a novel system for day-life applications.

\section{Discussion}

The metasurface arrangement, obtained using an inverse design mediated by deep machine learning (DML), provides the best configuration to deflect the light beam with a specific angle. In the proposed case, the deflection is not only determined by the metasurface arrangements as in an active configuration but is also assisted by the change of the surrounding medium. In fact, it passes from 1.52 to 1.92 by means of an external stimulus. In fact, by applying an external potential difference of few Volts the LC molecules change their orientation and as consequence the refractive index enabling the beam steering in the selected angle range. It is worth noting that the refractive index can be tuned continuously 
and hence each angle among the two extremes can be exploited. The investigated device is presented in Figure 1a, where three different colors indicate the LC orientations and relative refractive index. Figure $1 \mathrm{~b}$ illustrate a $2 \mathrm{D}$ view of the proposed device with the indication of each material thickness. The uniform ITO thickness of $50 \mathrm{~nm}$, even if is still transparent, ensures the driving of LC which has a thickness of $10 \mu \mathrm{m}$. By applying only $10 \mathrm{~V}$, a complete switching of LC is reached within $30-50 \mathrm{~ms}$ while the relaxation time is 200-300 ms. Hence the proposed design enables a very fast switching time and low operation voltage. A way to reduce the switching time is to use ferroelectric or dual-frequency liquid crystal $[29,30]$. Figure $1 \mathrm{c}$ reports a detailed sketch of the optimized metasurface with the indication of the deflection angle for different LC refractive index. In particular, in the absence of bias voltage, the corresponding angle is $45^{\circ}$, while by applying voltage it reaches $55^{\circ}$ or $65^{\circ}$. For validating the prediction obtained with the deep neural network and the optimization code, we simulate the behavior of final device when impinged by a Gaussian beam at $\lambda=1550 \mathrm{~nm}$ and the LC varies its refractive index. The simulations are performed using a home-built code written in python and using the diffraction library. The results are illustrated in Figure 2 where the $1550 \mathrm{~nm}$ transmitted beam position depends on the LC refractive index which passes from 1.52 to 1.92.

a)

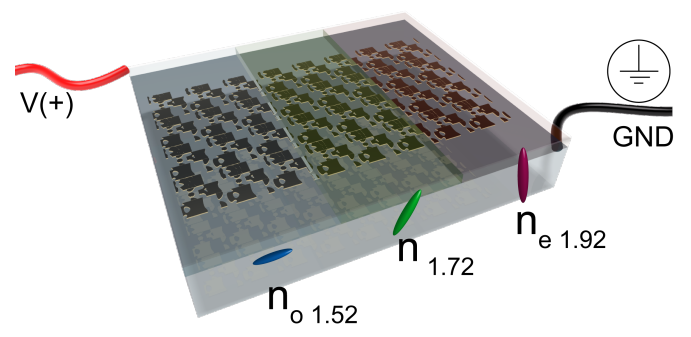

b)

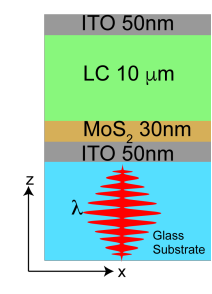

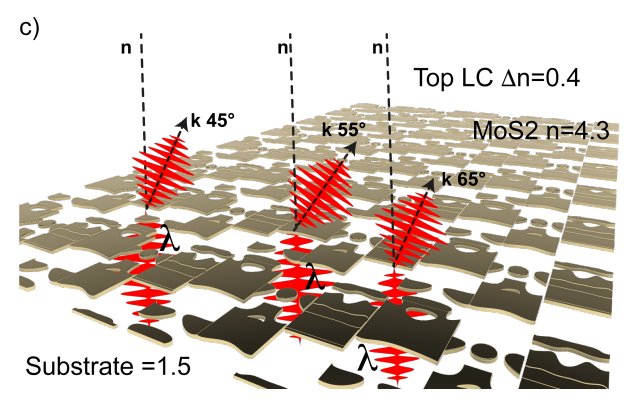

Figure 1. (a) Sketch of whole device composed of two ITO cover-slips containing the metastructures filled with the liquid crystal (LC). The ITO electrodes are connected to a FPGA processor that controls the applied voltage. (b) 2D Stack-up and relative thickness of each material. (c) A schematic view of the metasurfaces operation. The transmitted beam is deflected at the desired angle just by tuning the surrounding medium refractive index.

In order to clarify the operation mechanism of the proposed device, it is necessary to consider each involved element. The metastructures consist of a single thin film of $\mathrm{MoS}_{2}$ (thickness $30 \mathrm{~nm}$ ) with etched features, that in combination with substrate and LC can be generally treated as a vertically oriented Fabry-Perot cavity supporting a set of Bloch modes. In fact, the substrate-metasurface and metasurface-LC interfaces serve as cavity mirrors. The plane wave incident on the metasurface excites these modes, which bounce within the cavity. Whenever a mode interacts with a cavity mirror, a combination of three processes can occur, as described by coupled mode analysis [31-33]. First, the mode can reflect from the interface. Second, the mode can interact with and exchange energy with other modes. Third, the mode can scatter out of the cavity into several discrete diffraction channels. As such, each diffraction channel contains contributions from all of the modes. High deflection efficiency in the desired diffraction channel is achieved when the out-coupled plane waves from all the Bloch modes in that channel strongly 
constructively interfere. Multi-layered structures realized by two materials alternation are well investigated using the Bloch theory [34]. The Bloch mode supported by these devices elucidates the spatial mode profiles and coupling dynamics that make high-performance beam deflection possible.
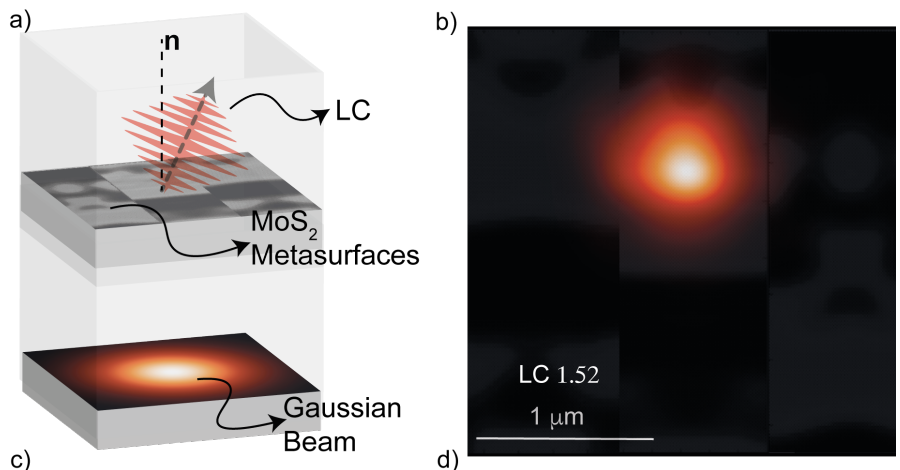

c) d)
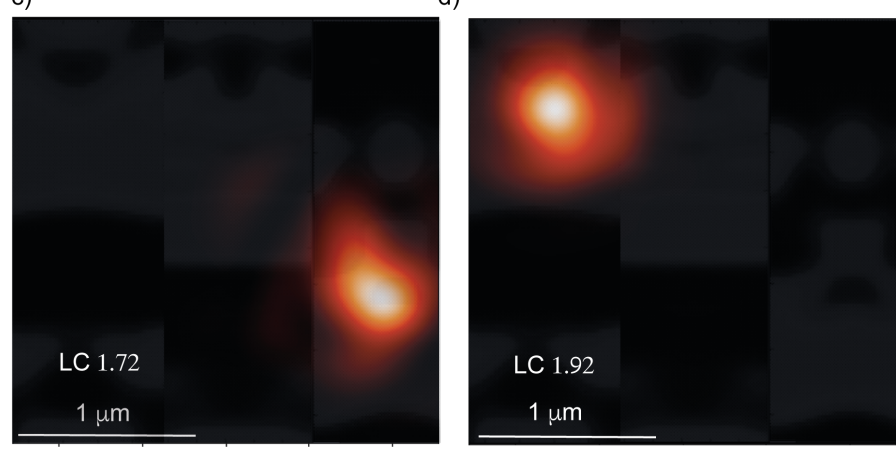

Figure 2. (a) Schematic illustration of the operation of the proposed device. (b-d) Numerical transmitted beam position for three different LC refractive index value.

In this work, the molybdenum disulfide $\left(\mathrm{MoS}_{2}\right)$ has been selected for its high refractive index in the near-infrared (NIR) spectrum, indeed at $\lambda=1550 \mathrm{~nm}$ its real part is 4.3 and its imaginary one is 0.034 that it could be negligible and the losses not affect the light beam. The complete device will be sandwiched among two cover-slips with a transparent, patterned and conductive layer (ITO) to drive the infiltrated liquid crystals. In fact, the ITO layers serve as electrodes and are connected to an FPGA control processor in order to mime the liquid crystal display functioning. The materials, constituting the device, have been selected because they are lossless in the NIR range and, last but not the least, the required sizes are not extremely sub-wavelength enabling an easy fabrication and integration. The deep machine learning tool used in this work is based on GLOnets algorithm $[35,36]$, on optimizer [37] and on convolutional neural network [38-40] and the working flow is schematically illustrated in Figure 3. The application of topology optimization to more complex nanophotonic devices originates from silicon photonic technology, and it has been applied to design other on-chip photonic devices [41], photonic crystals [42], and plasmonic absorbers [43]. Boundary optimization has been utilized to realize semiconducting absorbers [44] and optical sorters based on scalar diffraction [45]. The deep machine learning (DML) algorithm proposed in this manuscript-as already described —is based on the use of two Metanet repositories. In order to obtain the better configuration of the final device, the DML needs to receive as input the following parameters: the desired deflection angle (it could be a single value or a vector), the operating wavelength, the liquid crystals and $\mathrm{MoS}_{2}$ thickness (it could span in a vector e.g., from 10 to $100 \mathrm{~nm}$ ) and refractive index (real and imaginary part), see Figure 3. Then all of these parameters are stored in a large array that provides all possible combinations for the numerical system. Then, these data are used as input of the first neural network layer. 


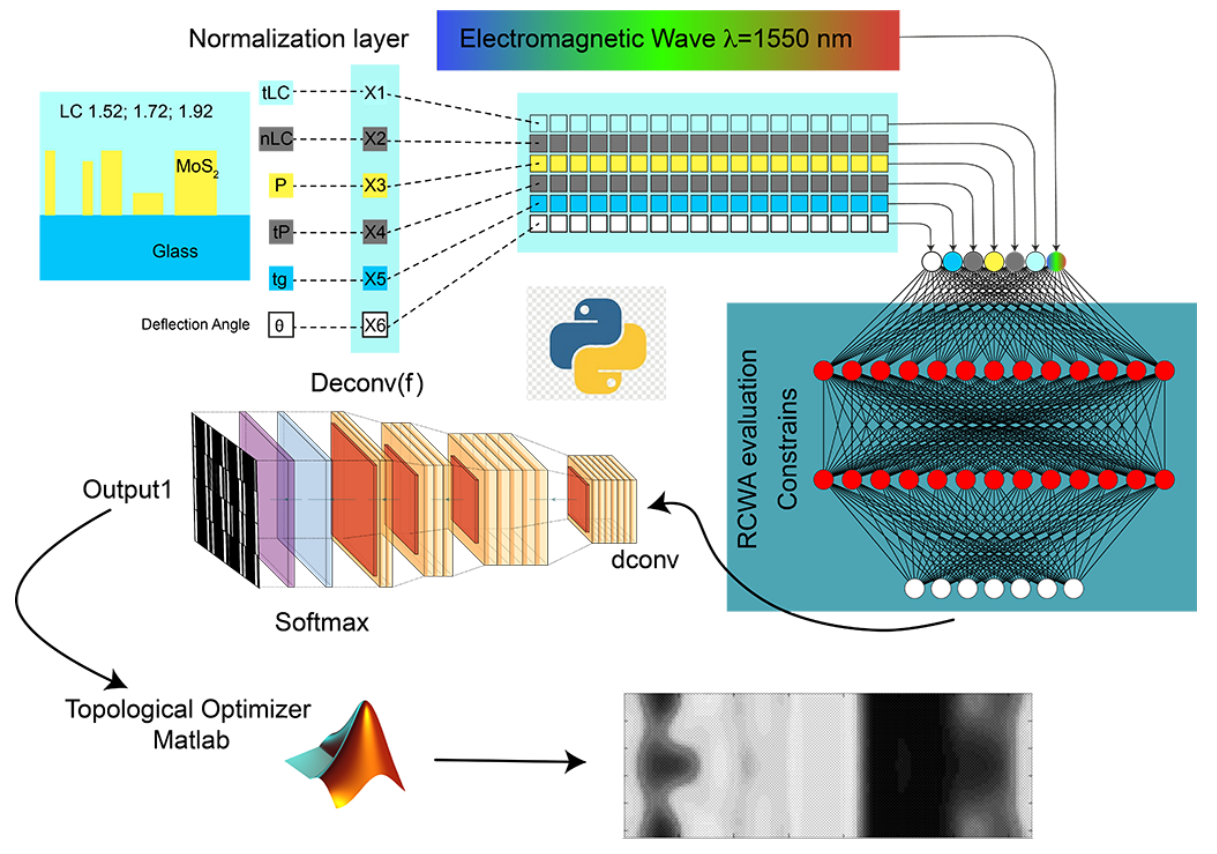

Figure 3. Working flow of the improved deep machine learning tool used to design the metasurfaces. The input data—such as material properties, number of elements, size-are stored and then processed in the neural network producing the input for the convolutional neural network that analyses the data and produces the preliminary $\mathrm{MoS}_{2}$ arrangements as output. The optimizer tool is involved to carry out the final structure pattern.

All the retrieved data are stored and a series of vectors are generated using all considerable combinations. Each data set is processed by the neural network that exploits a Rigorous Coupled Wave Analysis (RCWA) to evaluate the electromagnetic wave interaction with the metastructures. The algorithm is based on a global optimizer, based on a conditional generative neural network, which can output ensembles of highly efficient topology-optimized metasurfaces operating across a range of parameters, such as wavelength, desired deflection angle, size and geometry. A key feature of the network is that it initially generates a distribution of devices that broadly samples the design space and then shifts and refines this distribution toward favorable design space regions over the course of optimization. Training is performed by calculating, using the "reticolo code" written in Matlab [46] the forward and adjoint electromagnetic simulations of outputted devices and using the subsequent efficiency gradients for back-propagation. With structures that are similar to meta-gratings, the algorithm operates across a range of input parameters showing the devices, produced from the trained generative network, that have efficiencies comparable to or better than the best devices produced by adjoint-based topology optimization, while requiring a lower computational cost. This reframing of adjoint-based optimization to the training of a generative neural network is applied generally to physical systems that can utilize gradients to improve their performances $[35,36]$. Then, the modified GLOnets algorithms provide as output an attempt of working structure. By implementing and exploiting a Matlab optimizer it is possible to extrapolate the metasurfaces geometry able to maximize efficiency.

The metastructure shape is redefined in order to achieve the best one for the already specified wavelength and the deflection angle. The first one is to choose to modify the beam path with a deflection angle of $45^{\circ}$ dipped into an LC with a refractive index of 1.52, Figure $4 \mathrm{a}$. Then, the second one reflects the light with an angle of $55^{\circ}$ with $\mathrm{LC}$ refractive index equals 1.72 (Figure $4 \mathrm{~b}$ ), and finally, the last have to bend the light at $65^{\circ}$ when the surrounding medium $\mathbf{n}$ is 1.92 , see Figure $4 \mathrm{c}$. 

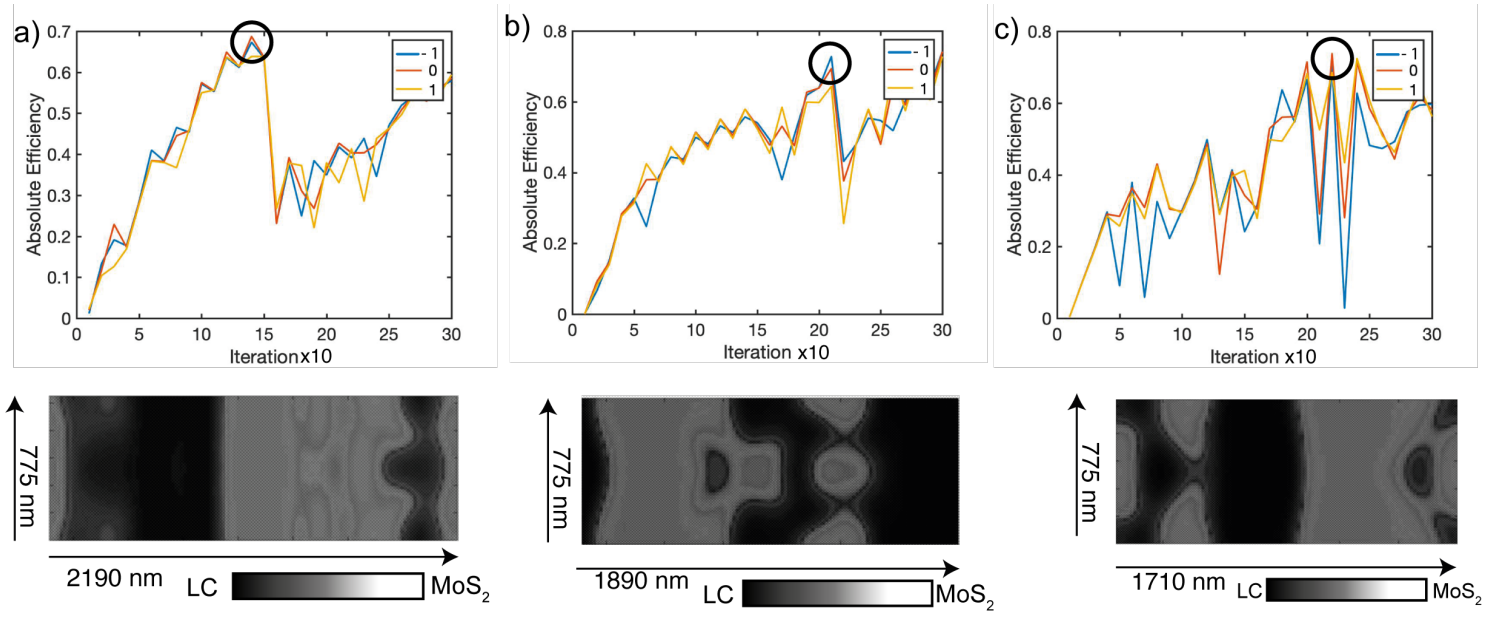

Figure 4. Illustration of the optimization steps for selecting the final structure pattern for the case (a) beam deflection of $45^{\circ}$ using the $n_{L C}$ equal to 1.52 (b) beam deflection of $55^{\circ}$ using the $n_{L C}$ equal to 1.72 and (c) beam deflection of $65^{\circ}$ using the $n_{L C}$ equal to 1.92. For each case is reported the efficiency plot and the corresponding structures for the best case indicated by the black circle in each plot.

\section{Conclusions}

In summary, the presented conceptual device, constituted of a tunable medium and an extremely optimized metasurface pattern arrangement, moves a leap forward for high control on the beam steering, and consequentially, the far-reaching implementation is reflected in the new LIDAR devices. The possibility to externally control the liquid crystal, and hence its refractive index, enable a continuous angle selection with a range depending on the birefringence of LC and on the metastructure design. This approach could represent a new paradigm in nano-photonic and nano-plasmonic mode engineering and utilizes a different physics approach from the current state-of-the-art, which is based on the stitching of noninteracting waveguide or grating structures. We believe that this method can envision that inverse design will enable new classes of high-performance photonic systems and new strategies toward the nanoscale control of light fields.

Author Contributions: Idea conceptualization G.E.L and A.F.; methodology, A.F.; software, G.E.L; validation, G.E.L and A.F.; formal analysis G.E.L and A.F.; writing-original draft preparation, G.E.L.; writing-review and editing, A.F.; visualization, G.E.L. and A.F.; supervision, A.F.; project administration, A.F. All authors have read and agreed to the published version of the manuscript. Authorship must be limited to those who have contributed substantially to the work reported.

Funding: This research received no external funding.

Institutional Review Board Statement: Not applicable, the reported studies not involving humans or animals.

Informed Consent Statement: Not applicable, The reported studies not involving humans.

Data Availability Statement: The data that support the findings of this study are available from the corresponding author upon reasonable request.

Acknowledgments: The authors thank "Sci-Design and Consulting" for the graphical artwork. The authors also thank the Infrastructure "BeyondNano" (PONa3-00362) of CNR-Nanotec for the access to research instruments. They thank the "Area della Ricerca di Roma 2", Tor Vergata, for the access to the ICT Services (ARToV-CNR), and the "METANET" numerical service and the related "GLOnets" and "Optimizer" tools kindly provided by Stanford University website.

Conflicts of Interest: The authors declare no conflict of interest. 


\section{References}

1. Wandinger, U. Introduction to lidar. In Lidar; Springer Series in Optical Sciences; Springer: New York, NY, USA, 2005; Volume 102; pp. 1-18.

2. Weitkamp, C. Lidar: Range-Resolved Optical Remote Sensing of the Atmosphere; Springer Science \& Business: New York, NY, USA, 2006; Volume 102; pp. 1-100.

3. Weiss, U.; Biber, P. Plant detection and mapping for agricultural robots using a 3D LIDAR sensor. Robot. Auton. Syst. 2011, 59, 265-273. [CrossRef]

4. Choi, J.; Ulbrich, S.; Lichte, B.; Maurer, M. Multi-target tracking using a 3d-lidar sensor for autonomous vehicles. In Proceedings of the 16th International IEEE Conference on Intelligent Transportation Systems (ITSC 2013), The Hague, The Netherlands, 6-9 October 2013; pp. 881-886. .

5. Infusino, M.; Ferraro, A.; De Luca, A.; Caputo, R.; Umeton, C. POLYCRYPS visible curing for spatial light modulator based holography. JOSA B 2012, 29, 3170-3176. [CrossRef]

6. Battal, E.; Okyay, A.K. Metal-dielectric-metal plasmonic resonators for active beam steering in the infrared. Opt. Lett. 2013, 38, 983-985. [CrossRef]

7. Forouzmand, A.; Mosallaei, H. Tunable two dimensional optical beam steering with reconfigurable indium tin oxide plasmonic reflectarray metasurface. J. Opt. 2016, 18, 125003. [CrossRef]

8. Wang, G.; Habib, U.; Yan, Z.; Gomes, N.J.; Sui, Q.; Wang, J.B.; Zhang, L.; Wang, C. Highly efficient optical beam steering using an in-fiber diffraction grating for full duplex indoor optical wireless communication. J. Light. Technol. 2018, 36, 4618-4625. [CrossRef]

9. Royo, S.; Ballesta-Garcia, M. An overview of lidar imaging systems for autonomous vehicles. Appl. Sci. 2019, 9, 4093. [CrossRef]

10. McManamon, P.F. Lidar Technologies and Systems; SPIE PRESS BOOK: Bellingham, WA, USA, 2019; Volume PM300, pp. 1-28

11. He, Q.; Sun, S.; Zhou, L. Tunable/reconfigurable metasurfaces: Physics and applications. Research 2019, 2019, 849272. [CrossRef]

12. Cui, T.; Bai, B.; Sun, H.B. Tunable metasurfaces based on active materials. Adv. Funct. Mater. 2019, 29, 1806692. [CrossRef]

13. Barna, V.; Caputo, R.; De Luca, A.; Scaramuzza, N.; Strangi, G.; Versace, C.; Umeton, C.; Bartolino, R.; Price, G.N. Distributed feedback micro-laser array: Helixed liquid crystals embedded in holographically sculptured polymeric microcavities. Opt. Express 2006, 14, 2695-2705. [CrossRef]

14. Zografopoulos, D.C.; Ferraro, A.; Beccherelli, R. Liquid-Crystal High-Frequency Microwave Technology: Materials and Characterization. Adv. Mater. Technol. 2019, 4, 1800447. [CrossRef]

15. Rocco, D.; Carletti, L.; Caputo, R.; Finazzi, M.; Celebrano, M.; De Angelis, C. Switching the second harmonic generation by a dielectric metasurface via tunable liquid crystal. Opt. Express 2020, 28, 12037-12046. [CrossRef]

16. Kudyshev, Z.A.; Kildishev, A.V.; Shalaev, V.M.; Boltasseva, A. Machine-learning-assisted metasurface design for high-efficiency thermal emitter optimization. Appl. Phys. Rev. 2020, 7, 021407. [CrossRef]

17. Ma, W.; Liu, Z.; Kudyshev, Z.A.; Boltasseva, A.; Cai, W.; Liu, Y. Deep learning for the design of photonic structures. Nat. Photonics 2020, 15, 77-90. [CrossRef]

18. Bayati, E.; Pestourie, R.; Colburn, S.; Lin, Z.; Johnson, S.G.; Majumdar, A. Inverse designed metalenses with extended depth of focus. ACS Photonics 2020, 7, 873-878. [CrossRef]

19. Li, Y.; Xu, Y.; Jiang, M.; Li, B.; Han, T.; Chi, C.; Lin, F.; Shen, B.; Zhu, X.; Lai, L.; et al. Self-Learning Perfect Optical Chirality via a Deep Neural Network. Phys. Rev. Lett. 2019, 123, 213902. [CrossRef]

20. Ashalley, E.; Acheampong, K.; Besteiro, L.V.; Yu, P.; Neogi, A.; Govorov, A.O.; Wang, Z.M. Multitask deep-learning-based design of chiral plasmonic metamaterials. Photonics Res. 2020, 8, 1213-1225. [CrossRef]

21. Lee, J.; Stanley, M.; Spanias, A.; Tepedelenlioglu, C. Integrating machine learning in embedded sensor systems for Internet-ofThings applications. In Proceedings of the 2016 IEEE International Symposium on Signal Processing and Information Technology (ISSPIT), Limassol, Cyprus, 12-14 December 2016; pp. 290-294.

22. Li, C.; Cao, X.; Wu, K.; Li, X.; Chen, J. Lens-based integrated 2D beam-steering device with defocusing approach and broadband pulse operation for Lidar application. Opt. Express 2019, 27, 32970-32983. [CrossRef]

23. Inoue, D.; Ichikawa, T.; Kawasaki, A.; Yamashita, T. Demonstration of a new optical scanner using silicon photonics integrated circuit. Opt. Express 2019, 27, 2499-2508. [CrossRef]

24. Wulfmeyer, V.; Bauer, H.; Di Girolamo, P.; Serio, C. Comparison of active and passive water vapor remote sensing from space: An analysis based on the simulated performance of IASI and space borne differential absorption lidar. Remote Sens. Environ. 2005, 95, 211-230. [CrossRef]

25. Burton, S.; Ferrare, R.A.; Hostetler, C.; Hair, J.; Kittaka, C.; Vaughan, M.; Obland, M.; Rogers, R.; Cook, A.; Harper, D.; et al. Using airborne high spectral resolution lidar data to evaluate combined active plus passive retrievals of aerosol extinction profiles. $J$. Geophys. Res. Atmos. 2010, 115. [CrossRef]

26. Jansson, S.; Brydegaard, M. Passive kHz lidar for the quantification of insect activity and dispersal. Anim. Biotelem. 2018, 6, 6. [CrossRef]

27. Viheriälä, J.; Aho, A.T.; Uusitalo, T.; Lyytikäinen, J.; Hallman, L.; Ryvkin, B.S.; Avrutin, E.A.; Kostamovaara, J.T.; Guina, M. High-power $1.5 \mu \mathrm{m}$ laser diodes for LIDAR applications. In Proceedings of the 2019 IEEE High Power Diode Lasers and Systems Conference (HPD), Coventry, UK, 9-10 October 2019; pp. 9-10. [CrossRef]

28. Dąbrowski, R.; Kula, P.; Herman, J. High birefringence liquid crystals. Crystals 2013, 3, 443-482. [CrossRef] 
29. Guo, Q.; Zhao, X.; Zhao, H.; Chigrinov, V. Reverse bistable effect in ferroelectric liquid crystal devices with ultra-fast switching at low driving voltage. Opt. Lett. 2015, 40, 2413-2416. [CrossRef] [PubMed]

30. Kowerdziej, R.; Wróbel, J.; Kula, P. Ultrafast electrical switching of nanostructured metadevice with dual-frequency liquid crystal. Sci. Rep. 2019, 9, 20367. [CrossRef]

31. Botten, I.; Craig, M.; McPhedran, R.; Adams, J.; Andrewartha, J. The dielectric lamellar diffraction grating. Opt. Acta Int. J. Opt. 1981, 28, 413-428. [CrossRef]

32. Lalanne, P.; Hugonin, J.P.; Chavel, P. Optical properties of deep lamellar gratings: A coupled Bloch-mode insight. J. Light. Technol. 2006, 24, 2442-2449. [CrossRef]

33. Lio, G.E.; Ferraro, A.; Giocondo, M.; Caputo, R.; De Luca, A. Color Gamut Behavior in Epsilon Near-Zero Nanocavities during Propagation of Gap Surface Plasmons. Adv. Opt. Mater. 2020, 8, 2000487. [CrossRef]

34. Maccaferri, N.; Isoniemi, T.; Hinczewski, M.; Iarossi, M.; Strangi, G.; De Angelis, F. Designer Bloch plasmon polariton dispersion in grating-coupled hyperbolic metamaterials. APL Photonics 2020, 5, 076109. [CrossRef]

35. Jiang, J.; Fan, J.A. Global optimization of dielectric metasurfaces using a physics-driven neural network. Nano Lett. 2019, 19, 5366-5372. [CrossRef] [PubMed]

36. Jiang, J.; Fan, J.A. Simulator-based training of generative neural networks for the inverse design of metasurfaces. Nanophotonics 2019, 9. [CrossRef]

37. Sell, D.; Yang, J.; Doshay, S.; Yang, R.; Fan, J.A. Large-angle, multifunctional metagratings based on freeform multimode geometries. Nano Lett. 2017, 17, 3752-3757. [CrossRef]

38. Tabian, I.; Fu, H.; Sharif Khodaei, Z. A convolutional neural network for impact detection and characterization of complex composite structures. Sensors 2019, 19, 4933. [CrossRef] [PubMed]

39. Yeung, C.; Tsai, J.M.; King, B.; Kawagoe, Y.; Ho, D.; Knight, M.W.; Raman, A.P. Elucidating the Behavior of Nanophotonic Structures through Explainable Machine Learning Algorithms. ACS Photonics 2020, 7, 2309-2318. [CrossRef]

40. Wu, Z.; Zhou, M.; Khoram, E.; Liu, B.; Yu, Z. Neuromorphic metasurface. Photonics Res. 2020, 8, 46-50. [CrossRef]

41. Piggott, A.Y.; Lu, J.; Lagoudakis, K.G.; Petykiewicz, J.; Babinec, T.M.; Vučković, J. Inverse design and demonstration of a compact and broadband on-chip wavelength demultiplexer. Nat. Photonics 2015, 9, 374-377. [CrossRef]

42. Lin, Z.; Pick, A.; Lončar, M.; Rodriguez, A.W. Enhanced spontaneous emission at third-order Dirac exceptional points in inverse-designed photonic crystals. Phys. Rev. Lett. 2016, 117, 107402. [CrossRef]

43. Fu, S.M.; Zhong, Y.K.; Ju, N.P.; Tu, M.H.; Chen, B.R.; Lin, A. Broadband polarization-insensitive metamaterial perfect absorbers using topology optimization. IEEE Photonics J. 2016, 8, 1-11. [CrossRef]

44. Sheng, X.; Johnson, S.G.; Michel, J.; Kimerling, L.C. Optimization-based design of surface textures for thin-film Si solar cells. Opt. Express 2011, 19, A841-A850. [CrossRef]

45. Xiao, T.P.; Cifci, O.S.; Bhargava, S.; Chen, H.; Gissibl, T.; Zhou, W.; Giessen, H.; Toussaint, K.C., Jr.; Yablonovitch, E.; Braun, P.V. Diffractive spectral-splitting optical element designed by adjoint-based electromagnetic optimization and fabricated by femtosecond 3D direct laser writing. ACS Photonics 2016, 3, 886-894. [CrossRef]

46. Hugonin, J.P.; Lalanne, P. Reticolo software for grating analysis. arXiv 2005, arXiv:2101.00901. 